\title{
Transfusion status in liver and kidney transplantation recipients: results from nationwide claims database
}

\author{
Dong Ho Choi ${ }^{1}$, Boyoung Park², Junghyun Yoon ${ }^{2}$, Han Joon Kim¹, Yun Kyung Jung ${ }^{1}$, Kyeong Geun Lee \\ ${ }^{1}$ Division of Hepatobiliary, Department of Surgery, Hanyang University Medical Center, Seoul, Korea \\ ${ }^{2}$ Department of Preventive Medicine, Hanyang University College of Medicine, Seoul, Korea
}

Background: This study analyzed the status and trends of transfusion and its associated factors among liver and kidney transplantation recipients.

Methods: A total of 10,858 and 16,191 naïve liver or kidney transplantation recipients from 2008 to 2017 were identified through the National Health Insurance Service database. The prescription code for transfusion and the presence, number, and amount of each type of transfusion were noted. The odds ratios and $95 \%$ confidence intervals were determined to identify significant differences in transfusion and blood components by liver and kidney transplantation recipient characteristics.

Results: In this study, $96.4 \%$ of liver recipients and $59.7 \%$ of kidney recipients received transfusions related to the transplantation operation, mostly platelet and fresh frozen plasma. Higher perioperative transfusion in women and declining transfusion rates from 2008 to 2017 were observed in both liver and kidney recipients. In liver recipients, the transfusion rate in those who received organs from deceased donors was much higher than that in those who received organs from living donors; however, the mortality rate according to transfusion was higher in recipients of deceased donor organs. In kidney recipients, a higher mortality rate was observed in those receiving transfusion than that in patients without transfusion.

Conclusions: In Korea, the transfusion rates in liver and kidney recipients were relatively higher than those in other countries. Sociodemographic factors, especially sex and year of transplantation, were associated with transfusion in solid organ recipients, possibly as surrogates for other causal clinical factors.

Corresponding author: Dong Ho Choi

E-mail: crane87@hanyang.ac.kr

(c) The Korean Society for Transplantation

This is an Open Access article distributed under the terms of the Creative Commons Attribution Non-Commercial License (http://creativecommons.org/licenses/by-nc/4.0/) which permits unrestricted non-commercial use, distribution, and reproduction in any medium, provided the original work is properly cited. 